LAWRENCE LIVERMORE N A T IO N A L LABORATORY
Application of Novel High Order Time
Domain Vector Finite Element Method to
Photonic Band-Gap Waveguides

R. Rieben, D. White, G. Rodrigue

January 14, 2004

2004 IEEE Antennas and Propagation Symposium Monterey, CA, United States

June 20, 2004 through June 26, 2004 
This document was prepared as an account of work sponsored by an agency of the United States Government. Neither the United States Government nor the University of California nor any of their employees, makes any warranty, express or implied, or assumes any legal liability or responsibility for the accuracy, completeness, or usefulness of any information, apparatus, product, or process disclosed, or represents that its use would not infringe privately owned rights. Reference herein to any specific commercial product, process, or service by trade name, trademark, manufacturer, or otherwise, does not necessarily constitute or imply its endorsement, recommendation, or favoring by the United States Government or the University of California. The views and opinions of authors expressed herein do not necessarily state or reflect those of the United States Government or the University of California, and shall not be used for advertising or product endorsement purposes. 


\title{
Application of Novel High Order Time Domain Vector Finite Element Method to Photonic Band-Gap Waveguides
}

\author{
R. Rieben, D. White, G. Rodrigue \\ Lawrence Livermore National Laboratory ${ }^{\dagger}$
}

\section{Introduction}

In this paper we motivate the use of a novel high order time domain vector finite element method that is of arbitrary order accuracy in space and up to 5th order accurate in time; and in particular, we apply it to the case of photonic band-gap (PBG) structures. Such structures have been extensively studied in the literature with several practical applications; in particular, for the low loss transmission of electromagnetic energy around sharp 90 degree bends [1]. Typically, such structures are simulated via a numerical solution of Maxwell's equations either in the frequency domain or directly in the time domain over a computational grid. The majority of numerical simulations performed for such structures make use of the widely popular finite difference time domain (FDTD) method [2], where the time dependent electric and magnetic fields are discretized over a "dual" grid to second order accuracy in space and time. However, such methods do not generalize to unstructured, non-orthogonal grids or to higher order spatial discretization schemes. To simulate more complicated structures with curved boundaries, such as the structure of [3], a cell based finite element method with curvilinear elements is preferred over standard stair-stepped Cartesian meshes; and to more efficiently reduce the effects of numerical dispersion, a higher order method is highly desirable [4]. In this paper, the high order basis functions of [5] are used in conjunction with the high order energy conserving symplectic time integration algorithms of [6] resulting in a high order, fully mimetic, mixed vector finite element method.

There are several other advantages that can be obtained by employing a high order time domain vector finite element method for the simulation of PBG waveguides. For open region problems that employ the Maxwellian perfectly matched layer (PML) technique of [7], use of higher order methods can lead to improved computational performance. Typically, the absorbing PML region is defined by a series of first order elements which approximate a rise in electric and magnetic conductivity defined by a third order polynomial. Using third order polynomial basis functions, we can reduce the PML region to a single layer by defining appropriate cubic tensor functions of space, then "projecting" these functions over the single element PML layer. In addition, high order energy conserving time integration methods can yield better performance in the conservation of numerical energy for the same computational cost as a standard low order method (such as the second order accurate leap-frog method).

\section{Discrete Ampere-Faraday Equations}

We begin with the coupled first order time dependent Maxwell equations

$$
\begin{aligned}
\epsilon \frac{\partial}{\partial t} \mathbf{E} & =\nabla \times\left(\mu^{-1} \mathbf{B}\right)-\sigma \mathbf{E}-\mathbf{J}(t) \\
\frac{\partial}{\partial t} \mathbf{B} & =-\nabla \times \mathbf{E}-\sigma^{*}\left(\mu^{-1} \mathbf{B}\right)
\end{aligned}
$$

where $\epsilon$ and $\mu$ denote dielectric and magnetic material property functions while $\sigma$ and $\sigma^{*}$ denote electric and magnetic conductivity functions (the fictitious magnetic conductivity is added to facilitate the Maxwellian PML). Each of the these functions is free to be a symmetric tensor function of space. Using a Galerkin finite element procedure with 1-form (or Curl-conforming) vector basis functions to discretize the electric field intensity and 2-form (or Div-conforming) vector

\footnotetext{
*rieben1@llnl.gov

$\dagger^{\dagger}$ This work was performed under the auspices of the U.S. Department of Energy by the University of California, Lawrence Livermore National Laboratory under contract No. W-7405-Eng-48, UCRL-PROC201827.
} 
basis functions to discretize the magnetic flux density, and assuming a relative free space magnetic permeability (i.e. $\mu^{-1}=1$ ) yields the following linear system of ODE's

$$
\begin{aligned}
M_{\epsilon} \frac{\partial}{\partial t} e & =K^{T} M_{\mu} b-M_{\sigma} e-M_{\epsilon} j \\
\frac{\partial}{\partial t} b & =-K e-M_{\sigma^{*}} b
\end{aligned}
$$

where $e$ and $b$ represent the discrete differential 1-form and 2-form electric and magnetic fields respectively, $K$ is a rectangular matrix representing the discrete curl operator and $j$ is the discrete 2 -form time dependent current source. The various $M_{*}$ are symmetric positive definite (SPD) mass matrices computed using the appropriate basis functions and material property functions. Note that the vectors $e$ and $b$ will have different dimensions and that the matrix $K$ will be rectangular. This is due to the dimensions of the Nédeléc polynomial spaces from which they are derived [8]. In this particular finite element method the instantaneous electromagnetic energy is the numerical version of the total energy given by

$$
\mathcal{E}=e^{T} M_{\epsilon} e+b^{T} M_{\mu} b
$$

Many time integration methods such as forward Euler, backward Euler, Runge-Kutta, AdamsBashforth, etc. are inherently dissipative and the energy as measured by (3) is not conserved; given an initial condition the electromagnetic energy will decay exponentially. However, as shown in [6] the use of symplectic integrators for the ODEs of (2) results in time averaged energy conservation. In addition, higher order methods excel in reducing the effects of numerical dispersion. An analytical dispersion analysis is only tractable for $2 \mathrm{D}$ meshes [4], here we present computational results that clearly demonstrate reduced numerical dispersion in 3D. Consider the propagation of a sinusoidally driven EM wave down a coaxial waveguide that spans several wavelengths (i.e. an electrically large problem). For a fixed mesh size, our novel high order method at 4th order accuracy can reduce the effects of numerical dispersion by up to 2 orders of magnitude in contrast to standard low order methods as shown in Figure 1.

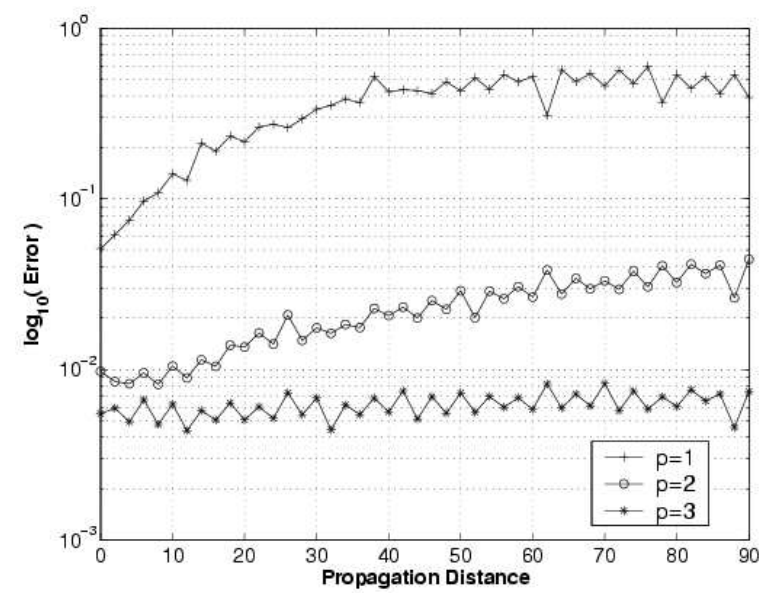

Figure 1: $L_{2}$ norm of computed arror as a function of propagation distance down a fixed coaxial waveguide mesh using basis functions of degree 1,2 and 3.

\section{Computational Results}

Here we simulate the propagation of an optical signal around a sharp 90 degree bend in a three dimensional slab PBG waveguide using high order time domain vector finite element methods. The PBG structure consists of a 9 by 9 array of Gallium-Arsenide (GaAs) cylinders oriented normal to the propagation plane, with a relative dielectric permittivity of $\epsilon_{r}=12.0$, surrounded by a square section of air $\left(\epsilon_{r}=1.0\right)$. Periodic structures such as these are characterized by the ratio of the rod radius $r$ to the rod spacing, or lattice constant, $a$. For the computational mesh used in these simulations, the ratio $\frac{r}{a}=0.18$ is chosen for a frequency of $\lambda=1.55 \mu \mathrm{m}$, this gives a transverse 
magnetic (TM) band-gap of $\omega=0.302 \frac{2 \pi c}{a}$ to $\omega=0.443 \frac{2 \pi c}{a}$. The rod spacing for the geometry is $a=0.62 \mu \mathrm{m}$. A waveguide can be constructed by removing some of the cylinders and introducing a defect; allowing a small range of wavelengths of light around a central defect frequency to propagate through the structure.

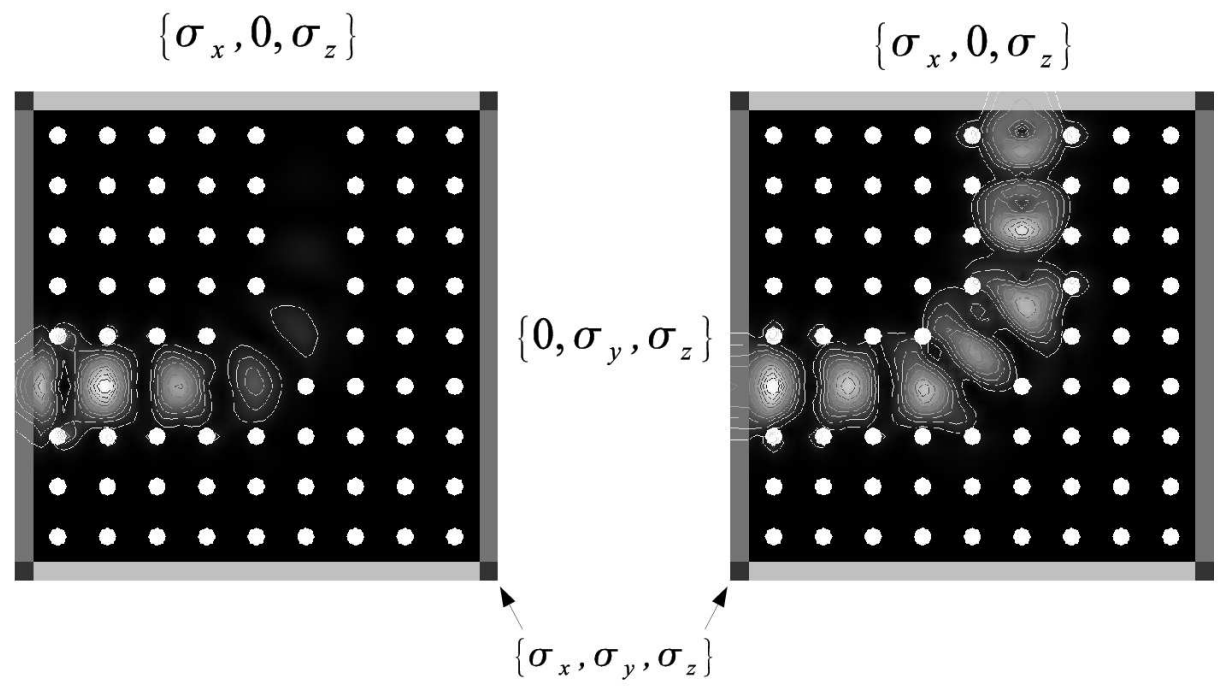

Figure 2: Magnitude of transmitted power in PBG waveguide with single element thick PML region using a sinusoidally driven voltage source at $t=0.042 \mathrm{ps}$ (left) and $t=0.084$ ps (right).

Figure 2 shows the results of a PBG waveguide simulation using third order interpolatory polynomial 1-form and 2-form basis functions. The problem is excited with a sinusoidally driven voltage source applied on the left hand side of the mesh. To account for the open region boundary, the computational domain is surrounded by a single element thick Maxwellian PML. The PML is divided into three regions, the $y$-dependent sides, the $x$-dependent top and bottom and the $x y$ dependent corners. An isotropic tensor valued electric and magnetic conductivity profile is defined for each region. In each case, the conductivity profile is a third-order polynomial function of space.
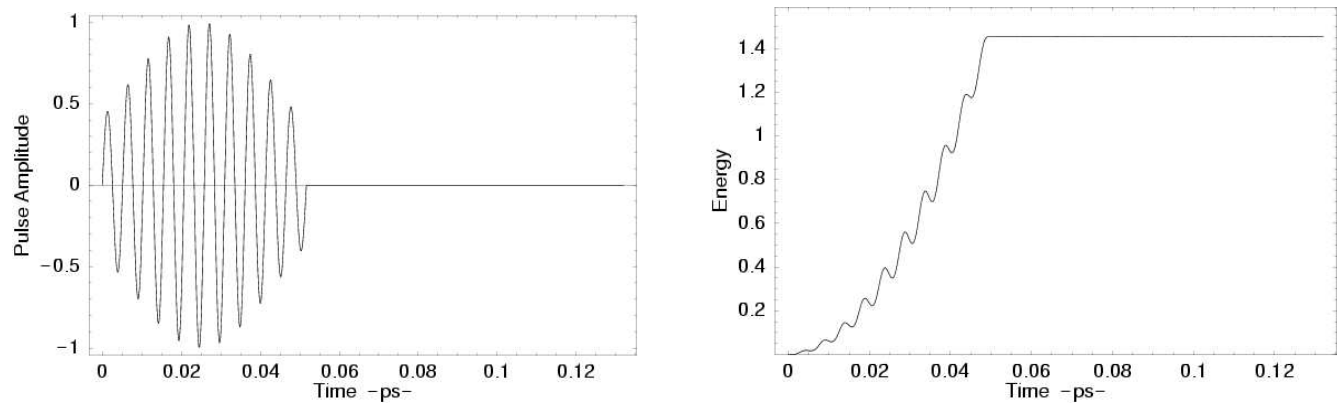

Figure 3: Temporal profile of pulsed voltage source (left) and numerical energy as a function of time for second order leap frog method (right).

Now if we neglect the electric and magnetic conductivity terms of (1) (i.e. $\sigma, \sigma^{*}=0$ ), the numerical energy of the problem as measured by (3) should be conserved. We now excite the PBG waveguide with a pulsed voltage source and deposit a finite amount of energy into the problem. The pulse has a temporal length equal to 10 wavelengths as shown in Figure 3. The resulting numerical energy as computed with the second order accurate and energy conserving leap-frog method is also shown in Figure 3. As expected, the energy ramps up until the pulse is switched off, at which point it remains constant for the duration of the simulation. In Figure 4, we show close ups of the computed numerical energy during the last half of the simulation for two different energy 

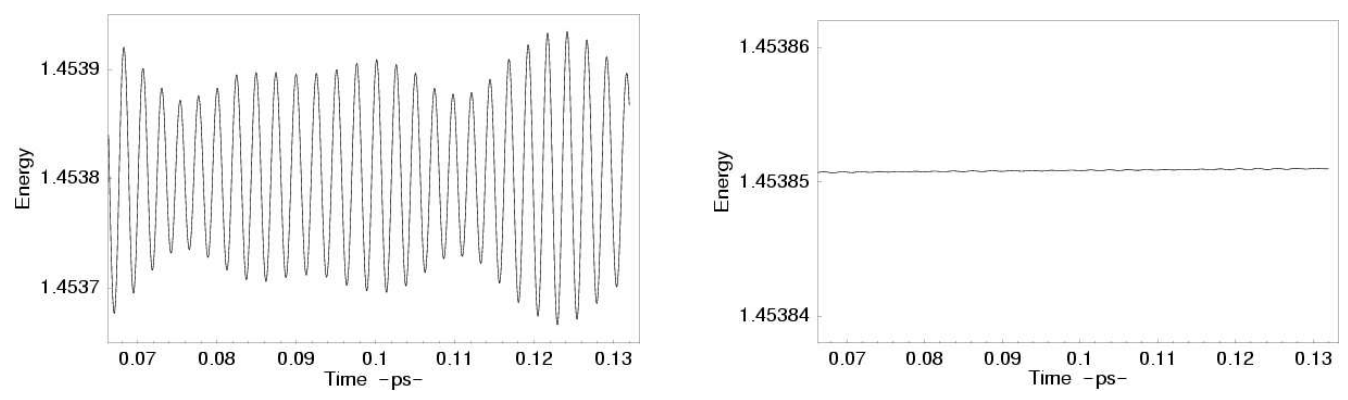

Figure 4: Close-up of numerical energy during last half of simulation for second order symplectic (or leap-frog) method (left) and fourth order symplectic method (right).

conserving integration methods: the standard second order accurate leap-frog method and a fourth order accurate symplectic integration method. For the second order method, a discrete time step of $\Delta t=0.0033333$ is used and the 1-form mass matrix $M_{\epsilon}$ of (2) needs to be solved once per time step, for a total of 12,000 time steps. For the fourth order method, a discrete time step of $\Delta t=0.01$ is used and the 1-form mass matrix $M_{\epsilon}$ of (2) needs to be solved three times per time step, for a total of 4000 time steps. The resulting computations therefore require the same total amount of time to complete. In both cases, the computed numerical energy will oscillate; however, as shown in Figure 4, the fourth order accurate method is much better at controlling the amplitude of this oscillation, for the same computational cost as the second order method.

\section{Conclusions}

We have applied the high order time domain vector finite element methods described in [5] and [6] to the particular case of photonic band-gap structures. We have demonstrated key advantages to such methods when applied to these structures; namely, the ability to use single element thick PML regions and the benefits of high order energy conserving time integration methods. In addition to these benefits, high order vector finite element methods excel at modeling geometries defined by non-orthogonal, unstructured grids and at reducing the effects of numerical dispersion when compared to traditional second order accurate FDTD and FE methods.

\section{References}

[1] A. Mekis, J. Chen, S. Fan, P. Villeneuve, and J. Joannopoulos. High transmission through sharp bends in photonic crystal waveguides. Phys. Rev. Let., 77(18):3787-3790, 1996.

[2] K. S. Yee. Numerical solution of initial boundary value problems involving Maxwell's equations in isotropic media. IEEE Trans. Ant. Prop., 14(3):302-307, 1966.

[3] E. Lidorikis, M. Povinelli, S. Johnson, and J. Joannopoulos. Polarization-independent linear waveguides in 3D photonic crystals. Phys. Rev. Let., 91(2), 2003. Art. No. 023902.

[4] M. Ainsworth and J. Coyle. Hierarchic $h p$-edge element families for Maxwell's equations on hybrid quadrilateral/triangular meshes. Comput. Methods Appl. Mech. Engrg., 190:6709-6733, 2001.

[5] R. Rieben, D. White, and G. Rodrigue. Improved conditioning of finite element matrices using new high order interpolatory bases. IEEE Trans. Ant. Prop., 2004. In press.

[6] R. Rieben, D. White, and G. Rodrigue. High order symplectic integration methods for finite element solutions to time dependent maxwell equations. IEEE Trans. Ant. Prop., August 2004. In press.

[7] R. Ziolkowski. The design of Maxwellian absorbers for numerical boundary conditions and for practical applications using engineered artificial materials. IEEE Trans. Ant. Prop., 45(4):656671, 1997.

[8] J. C. Nédélec. Mixed finite elements in R3. Numer. Math., 35:315-341, 1980. 